\title{
Gestión de cuencas en la región Metropolitana de Buenos Aires. Historia y actualidad de un territorio en conflicto ambiental. El caso del Gran La Plata
}

Daniela Vanesa Rotger

Arquitecta UNLP. Doctora en Arquitectura y Urbanismo (UNLP). Magíster en Paisaje, Medioambiente y Ciudad (FAUUNLP). Becaria posdoctoral CONICET en el Centro de Investigaciones Urbanas y Territoriales (CIUT-FAU-UNLP). Docente desde 2009 en el área de planificación territorial (cátedra López-Rocca-Etulain) en la facultad de Arquitectura de la Universidad de La Plata. rotgerdaniela@hotmail.com.

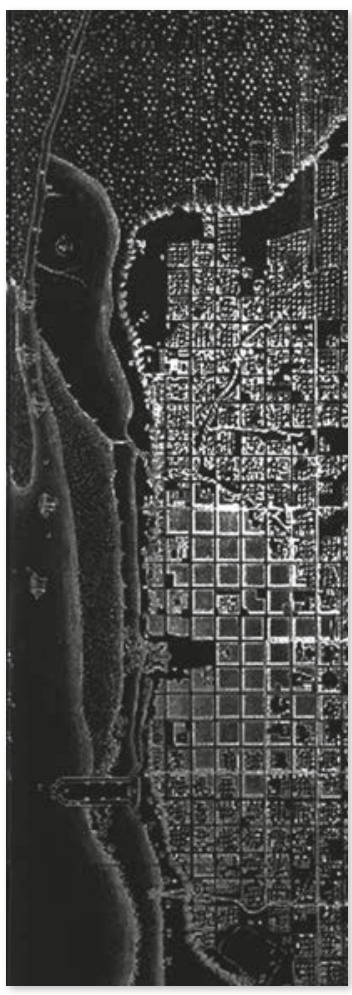




\title{
Resumen
}

La Región Metropolitana de Buenos Aires se desarrolla sobre un sistema de cuencas de escasa pendiente que desemboca en el río de La Plata. Al situarse en este territorio la mayor densidad poblacional del país, se plantean problemáticas ambientales relativas a las difíciles condiciones de escurrimiento, la adaptación de la urbanización al medio natural y la degradación de los cursos. ¿Cómo se ha construido a lo largo de la historia la relación entre medio natural y urbanización en la región? ¿Cómo se ha ido incorporando el tema ambiental en el ordenamiento territorial? ¿Cuál es el tratamiento actual de las cuencas? Son interrogantes que intenta responder este artículo, puntualizando el análisis en la cuenca más poblada del Gran La Plata, la del arroyo del Gato.

Para finalizar se identifican puntos críticos de cara a una gestión territorial que tome en cuenta la complejidad de las cuencas de la RMBA en materia ambiental.

\section{Palabras clave}

Gestión; cuencas; RMBA; arroyo del Gato.

\begin{abstract}
Watershed Management in the Metropolitan Region of Buenos Aires. History and Current Reality of a Territory in Environmental Conflict. The Case of Greater La Plata.

The Metropolitan Region of Buenos Aires developed upon a system of flatland watersheds of slow drainage that flow into the River Plate. Given that this territory has the highest density of population in Argentina, the difficult drainage conditions have resulted in many environmental problems complicating the urbanization process and resulting in the degradation of water courses.

How has the relationship between the natural environment and urbanization in the region been resolved throughout history? How has the environmental dimension been incorporated into land use planning? And what is the current treatment of the original watersheds? These are the questions that this article tries to answer, emphasizing its analysis in the most populated basin of the Greater La Plata region, the Del Gato stream. Finally, critical points are identified for territorial management that take into account the complexity of the environmental issues of the river basins of the Metropolitan Region of Buenos Aires (RMBA).
\end{abstract}

\section{Keywords}

Management; basins; RMBA; Del Gato Stream. 


\section{Introducción*}

Desde los aspectos que configuran el medio natural, la Región Metropolitana de Buenos Aires se caracteriza como un territorio predominantemente llano que se desarrolla en forma semicircular alrededor de la ciudad de Buenos Aires, extendiéndose a lo largo del estuario del río de La Plata, donde desemboca un sistema de cuencas de ríos y arroyos perpendiculares a la costa. Aunque la geografía regional no es particularmente compleja, la armonización entre el medio natural y el construido no es sencilla, considerando que se trata de un territorio ocupado por aproximadamente 15.000 .000 de habitantes, donde los ríos y arroyos discurren en sentido perpendicular al río de La Plata, mientras la urbanización se desarrolla en forma anular alrededor de la ciudad de Buenos Aires. Esto se evidencia en el impacto creciente de las inundaciones urbanas por precipitaciones, como problemática que ha adquirido mayor repercusión recientemente, además del fenómeno denominado sudestada, que afecta cíclicamente a la región, cuando los vientos procedentes del cuadrante sudeste impiden el normal escurrimiento de las aguas del río de La Plata.

Este artículo intenta poner en relieve los distintos enfoques que han incidido durante la historia urbana de la Región Metropolitana de Buenos Aires en el tratamiento de las cuencas, tanto los influidos desde la necesidad de uso y ocupación del territorio - y específicamente de las áreas inundables-que a lo largo del proceso de configuración del conglomerado incidieron en la afectación de los cursos y alteración del escurrimiento natural de las cuencas, como aquellos enfoques que desde el ordenamiento territorial han intentado dar respuesta a las problemáticas emergentes del proceso de urbanización. Específicamente se analiza el surgimiento del enfoque ambiental en el ordenamiento del territorio y en la gestión de cuencas desde mediados del siglo XX, momento en que comienza a manifestarse con claridad la discordancia entre naturaleza y ciudad en la región.

Siguiendo el correlato histórico, se plantea el estado actual en torno a la gestión de las cuencas y los principales avances vinculados con una visión más abarcadora del territorio, así como los importantes desafíos que impiden la integralidad necesaria en el tratamiento de esta unidad territorial. Brevemente se indagan estos temas en el Gran La Plata ${ }^{1}$ y en el arroyo del Gato, la cuenca más relevante de la capital provincial, tanto por su extensión como

\author{
* La investigación \\ desarrollada en el artículo \\ ha sido financiada por \\ fondos provenientes de una \\ beca interna posdoctoral \\ CONICET (Consejo \\ Nacional de Investigaciones \\ Científicas y Técnicas).
}

1. Conglomerado urbano formado por los partidos de La Plata, Berisso y Ensenada. 
2. La RMBA está conformada por los siguientes municipios: Alte. Brown, Avellaneda Berazategui, Berisso, Brandsen, Campana, Cañuelas, Ensenada,

Escobar, Esteban Echeverría, Exaltación de la Cruz, Ezeiza, Fcio. Varela, Gral. Rodríguez, Gral. San Martín, Gral. Las Heras, Hurlingham, Ituzaingó, José C. Paz, La Matanza, La Plata, Lanús, Lomas de Zamora, Luján, Malvinas Argentinas, Marcos Paz, Merlo, Moreno, Morón, Pilar, Pte. Perón, Quilmes, San Fernando, San Isidro, San Miguel, San Vicente, Tigre, Tres de Febrero, Vicente López y Zárate.

3. Fuente: datos tomados de http://www. observatorioamba.org/ planes-y-proyectos/ rmba\#datos). por su densidad, afectada por problemáticas ambientales de gravedad, entre ellas haber sido una de las más perjudicadas en la inundación ocurrida en la localidad en abril de 2013.

A modo de cierre, se identifican los tópicos centrales en pos de un tratamiento de cuencas que tome en cuenta la complejidad ambiental de este territorio, de cara a la resolución de las problemáticas ambientales existentes, pero también sentando las bases de una visión territorial que supere la mirada centrada en el hecho urbano, y que de esta manera considere a la cuenca como unidad sintética de los procesos ambientales de la región, siendo especialmente relevante para el análisis de un territorio dominantemente urbano como el de la RMBA.

\section{Cuencas de la Región Metropolitana de Buenos Aires}

La denominación Región Metropolitana de Buenos Aires ${ }^{2}$ (RMBA) refiere a un área geográfica conformada por la Ciudad Autónoma de Buenos Aires, el Gran Buenos Aires y un conjunto de partidos aledaños, que incluyendo al Gran La Plata - formado por los partidos de La Plata, Berisso y Ensenada - totaliza cuarenta municipios. Conforma una extensa conurbación cuyo espacio periférico se ha urbanizado rápidamente en las últimas décadas, siguiendo los ejes de las nuevas autopistas. Según la información del último Censo Nacional de Población y Vivienda (2010), la región reúne una población de 14.839.746 habitantes ${ }^{3}$, poco más del $37 \%$ de la población del país.

La RMBA se asienta sobre una planicie apenas ondulada que se configura alrededor del estuario del río de La Plata. El territorio no presenta grandes accidentes geográficos; sin embargo, está condicionado por su relación con el río de La Plata y las cuencas hidrográficas que definen franjas perpendiculares a la costa, y que en su discurrir desde la naciente hasta la desembocadura, atraviesan progresivamente una densidad poblacional mayor, lo cual repercute en la forma en que históricamente la sociedad se ha relacionado con el agua, experimentando determinadas "catástrofes naturales" cuando su ciclo entra en desajuste con el medio construido (Di PACE y BARSKY, 2012).

La región se implanta sobre cinco grandes cuencas hidrográficas perpendiculares al río de La Plata; de norte a sur se sitúan las cuencas del río Luján y Reconquista, la cuenca del río Matanza-Riachuelo hacia el suroeste, las cuencas de la Ciudad de Buenos Aires - con cursos totalmente entubados- - , por último, la cuenca de la zona sur (figura 1). 


\section{Gestión de cuencas en la región Metropolitana de Buenos Aires.}

\section{Historia y actualidad de un territorio en conflicto ambiental. El caso del Gran La Plata}

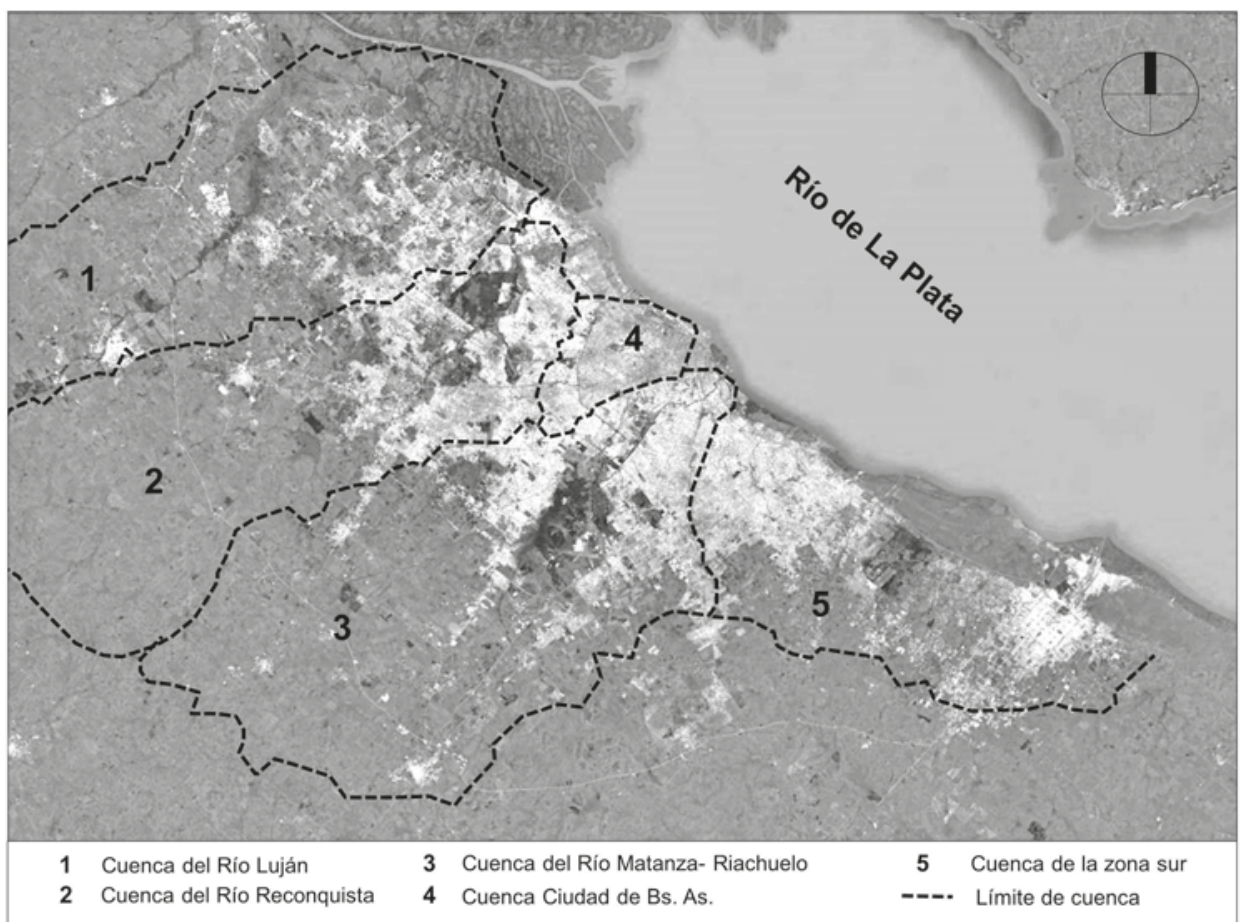

Todas estas cuencas, a pesar de poseer características territoriales comunes, tanto en relación con lo físico natural como con los patrones urbanos que afectan a la región como conglomerado, poseen diferencias en cuanto al uso y aprovechamiento del territorio, basadas en sus características particulares, las que motivan distinciones en cuanto a la valoración social y a la gestión del territorio. Las tres cuencas con mayor superficie de la región son, como ilustra el mapa, en sentido noroeste-sudeste las de los ríos Luján, Reconquista y $M a-$ tanza-Riachuelo. Les siguen las cuencas hidrográficas de la zona sur y las de la ciudad de Buenos Aires. De las tres cuencas mencionadas, la del río Luján es la que posee menor densidad poblacional - menos de un cuarto de la población que alberga la cuenca del río Re-

\section{Figura 1.}

Cuencas de la RMBA.

Fuente: elaboración propia sobre la base de imagen de Google Earth, 2017 
conquista, y menos de un tercio de la de la cuenca Matanza-Riachuelo-. La mayor parte de la cuenca corresponde a la zona de expansión más reciente de la región, en la que conviven centros urbanos de origen rural que se expandieron a partir de las nuevas autopistas; áreas agrícolas y urbanizaciones cerradas dispersas que se sitúan con mayor intensidad desde la localidad de Luján hasta la desembocadura en el río de La Plata, habiendo atravesado el paisaje deltaico que se desarrolla entre los partidos de San Isidro, San Fernando, Escobar y Tigre, y que suscita conflictos de intereses entre el uso público y privado de la ribera. El paisaje que genera el encuentro con el delta y el estuario del río de La Plata origina un ecotono en donde los valores paisajísticos favorecen el desarrollo de las lógicas de la urbanización cerrada (Fernández en Pintos y Narodowski, 2012).

Continuando hacia el sudeste, las cuencas de los ríos Reconquista y Matanza Riachuelo presentan características similares. A pesar de la mayor superficie de la primera y su menor densidad (aproximadamente 25 hab./ha y 15 hab./ha, respectivamente), ambas cuencas, al ubicarse en los límites norte y sur de la ciudad de Buenos Aires, poseen una gran densidad poblacional en las cuencas media y baja, en coincidencia con la primera y segunda corona de la Región Metropolitana de Buenos Aires, donde se produjo la mayor concentración de industrias desde la década de 1940, de lo cual derivaron el alto grado de contaminación actual de ambas cuencas y el surgimiento de sus respectivos planes de saneamiento.

A diferencia de la cuenca del río Reconquista, en la cuenca baja del Matanza-Riachuelo se asentaron las primeras industrias de la región, en vinculación con el puerto de Buenos Aires, dado que la llamada boca del Riachuelo funcionaba como puerto natural de la aglomeración y originaba además de un alto grado de contaminación desde hacía varios siglos, un vasto patrimonio cultural: relatos históricos, artículos periodísticos, ficciones literarias, fotografías y pinturas (SiLVESTRI, 2003), que se concentran en el tramo de su desembocadura.

Aunque no existan cursos fluviales visibles en la ciudad de Buenos Aires, pues se encuentran entubados, los cinco arroyos más importantes que surcan la ciudad son: Ugarteche, Los Terceros, Vega, Maldonado y Medrano (Herrero y Fernández, 2008). Por último, están las cuencas de la zona sur, frente formado por un sistema de arroyos que desaguan directamente en el río, aunque mediante canalizaciones en la planicie costera, donde los cursos se vuelven divagantes. La franja costera sur del río de la Plata se extiende desde el río Matanza-Riachuelo al norte, hasta los bañados de Maldonado al sur. 
En este sistema de cuencas, con diferente intensidad de ocupación —una mayor intensidad edificatoria entre la ciudad de Buenos Aires y el partido de Florencio Varela, notoriamente disminuida entre este partido y el Gran La Plata-, en general los cursos se encuentran muy transformados debido al uso predominantemente urbano de las cuencas medias. En las cuencas altas existen valores asociados al uso agrícola, y hacia la cuenca baja, sucede algo similar al delta y litoral de la cuenca Luján, en este caso con competencia de urbanizaciones cerradas, industrias, áreas de reserva natural en áreas de humedal y selva marginal, y también el uso de la ribera como espacio público.

El crecimiento metropolitano y la degradación ambiental que conlleva han ido en paralelo al deterioro de las cuencas de la RMBA. Históricamente la problemática que más se visibilizó fue la de la contaminación de las cuencas más urbanizadas, dado que ello es evidente y se manifiesta no solo en los cursos principales de los ríos y arroyos, sino en el río de La Plata como destinatario de los aportes de estas cuencas, ya que recibe contaminantes de diversas fuentes y tipos: bacterias coliformes fecales, metales pesados y plaguicidas, entre otros (SUBSECRETARÍA DE URBANismo y ViviENDA, 2007). Sin embargo, la problemática de las inundaciones comienza a cobrar cada vez más visibilidad, junto con una mayor conciencia de los temas ambientales, la expansión urbana sobre áreas frágiles y el incremento de episodios de precipitaciones de consideración, como el ocurrido el 2 de abril de 2013 en la ciudad de La Plata, cuando cayeron $392 \mathrm{~mm}$ en veinticuatro horas, lo que provocó el colapso de la capacidad de escurrimiento del sistema pluvial y el anegamiento de amplios sectores de la ciudad.

El próximo apartado intenta desentrañar cómo se ha dado el proceso de urbanización en relación con las cuencas de la región, poniendo en relieve los patrones históricos de uso y ocupación de las áreas inundables, así como las lógicas que incidieron en la gestión de este territorio.

\section{Breve historia de la urbanización sobre las cuencas de la RMBA}

El sitio elegido para la fundación de la ciudad de Buenos Aires ${ }^{4}$ cumplía con los requisitos de la fundación de ciudades en el siglo XVI, combinando un puerto natural (el Riachuelo) y un punto elevado como protección ante las crecidas. Se trataba de una planicie surcada por numerosos cursos fluviales de escasa pendiente y configuración meandriforme, y bordeada por una barranca elevada entre ocho y doce metros sobre la costa (GUTMAN y HARDOY, 2007).
4. Las dos fundaciones de Buenos Aires fueron emplazadas casi en el mismo sitio; solo existe entre ambas un kilómetro de distancia. 
Sin embargo, más allá de considerar la elevación de la ciudad como modo de protegerla ante las inundaciones, el recorrido sinuoso de los cursos no fue acompañado por la cuadrícula española, cuyas líneas rectas se superpusieron a los valles de inundación.

Sobre la base del parcelamiento colonial realizado por Garay en 1580, se fueron sucediendo una serie de oleadas aglomerativas, que han ido desarrollando la urbanización al compás de los procesos socioeconómicos (Di PACE y BaRSKY, 2012). En este sentido, Brailovsky (2010) identifica diferentes actitudes sociales y períodos respecto del fenómeno de las inundaciones urbanas en la ciudad de Buenos Aires y el Gran Buenos Aires, que dejan expuesta la relación entre el proceso de urbanización y el medio natural a lo largo de la historia de la Región Metropolitana de Buenos Aires. Según el autor citado, el período colonial (hasta 1810) se caracteriza por la escasa intervención del medio natural, tanto debido a los bajos índices demográficos como al incipiente desarrollo tecnológico.

Los bañados situados entre la barranca y la costa y los de los cursos de agua tenían expresa prohibición de ocupación y se consideraban como áreas "realengas" —es decir, de pertenencia al rey-, destinadas a la cría de ganado y al pastoreo, que más tarde fueron destinadas al uso agrícola y a la pesca para abastecimiento de alimentos a la ciudad. Asimismo las riberas y bajos también eran ocupados por sectores marginales de la ciudad, como los esclavos fugitivos. Algunos procesos degradantes del medio natural que comienzan a desarrollarse son la colmatación de los arroyos, debido a la deforestación y el aumento de la capacidad de arrastre de las aguas, así como el vertido de residuos domiciliarios en ellos.

Siguiendo a Brallovsky, en los primeros años de vida independiente (1810-1860), el crecimiento poblacional fue moderado, por lo que no existía tampoco una gran presión para ocupar las áreas inundables. A pesar de eso, comenzaron a formalizarse algunas de las ocupaciones de los bajos, por parte de pobladores de bajos recursos, en su mayoría pequeños productores agrícolas o pescadores. Desde el gobierno se dieron a lo largo de este período dos actitudes contrapuestas. Durante el gobierno de Rosas (1829-1852) comenzaron a instalarse saladeros sobre los valles de inundación de algunos arroyos. Por otro lado, durante el gobierno de Sarmiento (1868-1874) el bañado de Palermo - donde Rosas había construido su residencia - se convirtió en el parque 3 de Febrero. Siguiendo la línea de los parques de la ciudad de Buenos Aires, existía una idea de ocupar los bajos con espacios públicos para bloquear su poblamiento (BRAILovsky, 2010). 
Fue a fines del siglo XIX cuando comenzaron a registrarse transformaciones notorias en materia de paisaje y ambiente, con el impacto de la inmigración y la primera fase industrial: "se define una temprana organización metropolitana por la ubicación de crecimientos sobre los nuevos ejes del ferrocarril”" (SubsecretaRía de Urbanismo y Vivienda, 2007: 47). Los ejes principales de expansión son el eje oeste y sur de la RMBA, coincidiendo con las tierras altas entre las cuencas del río Reconquista y el Riachuelo.

El crecimiento metropolitano se produjo principalmente a partir de procesos de autoconstrucción alrededor de las industrias, las que fundamentalmente se concentraron en torno del Riachuelo. Esta etapa se caracteriza por una creciente intervención del medio natural, que intentará emular la dominación de la naturaleza ejercida en el continente europeo.

El avance de la propiedad privada y la confianza en la ciencia y tecnología diezmaron los espacios inundables de uso público. En esta época se aprobó la enajenación de bañados sobre el río de La Plata y el Paraná, se limitó el ancho de la franja pública al río de La Plata en su total extensión a 150 varas, y el código civil dispuso un ancho de ribera como Camino de Sirga de $35 \mathrm{~m}$, "el llamado camino de sirga que se usa para arrastrar los barcos desde la orilla, indispensable en la época de las embarcaciones a vela” (Brailovsky, 2010: 101). Asimismo, el crecimiento demográfico y la extensión de la urbanización hicieron que se perdiera el registro del medio natural en los mapas de la época (figura 2).

Los arroyos se fueron adaptando progresivamente a la urbanización, primero mediante la canalización -convirtiéndolos en conductos artificiales-, luego comenzaron a analizarse obras de entubamiento. Aunque el ferrocarril quedó a salvo de las inundaciones, los loteos populares fueron víctimas de sistemáticas inundaciones.

En una primera etapa del proceso de industrialización iniciado por la sustitución de importaciones (1930/1955), se produjo un crecimiento periférico extensivo por la instalación de las industrias en la segunda corona de la RMBA, la atracción de inmigración y la suburbanización de los trabajadores urbanos. Desde la década de 1930, pero sobre todo entre 1940 y 1950, este crecimiento demográfico se debió a migraciones internas (GuTMAn y HaRDor, 2007).

La subdivisión del suelo rural en pequeñas parcelas permitió el acceso masivo a la propiedad individual en áreas periféricas. En las zonas bajas de humedales y cuencas comienzan a 
Figura 2. Ciudad de Buenos Aires. Planos de 1782 y 1893. Fuente: Archivo digital del Gobierno de la Ciudad de Buenos Aires
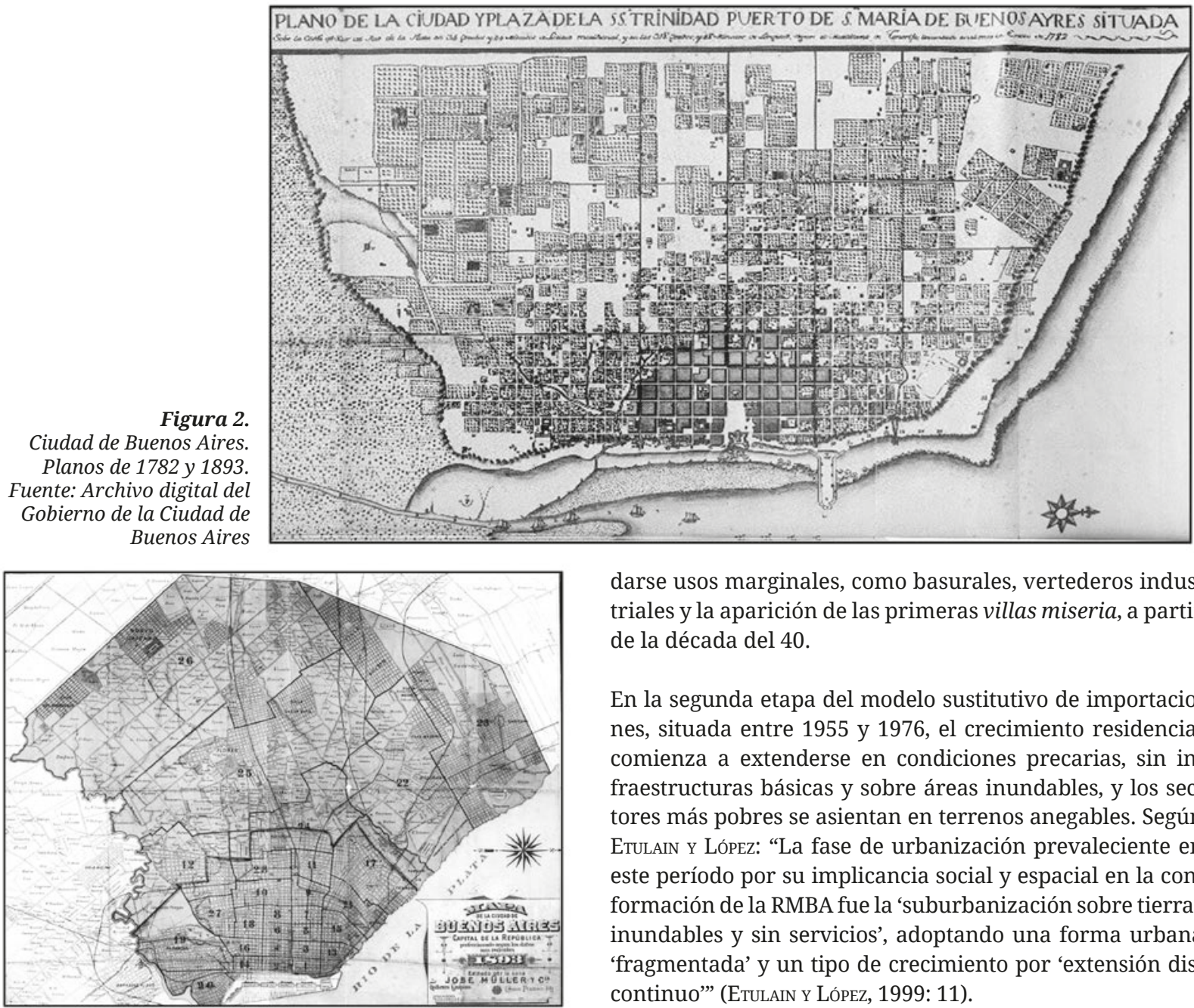

darse usos marginales, como basurales, vertederos industriales y la aparición de las primeras villas miseria, a partir de la década del 40.

En la segunda etapa del modelo sustitutivo de importaciones, situada entre 1955 y 1976, el crecimiento residencial comienza a extenderse en condiciones precarias, sin infraestructuras básicas y sobre áreas inundables, y los sectores más pobres se asientan en terrenos anegables. Según ETULAIN Y López: "La fase de urbanización prevaleciente en este período por su implicancia social y espacial en la conformación de la RMBA fue la 'suburbanización sobre tierras inundables y sin servicios', adoptando una forma urbana 'fragmentada' y un tipo de crecimiento por 'extensión discontinuo"” (Etulain y López, 1999: 11). 
Entre 1976 y 1991, con el modelo aperturista importador, la actividad industrial en la región es restringida y desalentada. La desocupación y la precarización de los salarios producen un aumento sustancial de la pobreza urbana, y se incrementan las villas y surgen otros hábitats informales sobre áreas anegables. El aumento de la pobreza en este período producirá un proceso de avance constante hasta la actualidad: el de poblaciones carenciadas asentadas social y físicamente sobre las orillas de los cursos de agua en condiciones de alta vulnerabilidad ambiental.

Así es como en la historia de la RMBA, por distintas razones, la urbanización se desarrolló omitiendo la dinámica del soporte natural, un proceso en el que la sociedad "construyó la ficción de que la artificialización del medio anula las leyes de la naturaleza” (BraiLovsky, 2010: 55). Sin embargo, esta negación no hizo más que exacerbar los efectos negativos de la problemática relación entre ciudad y cuencas, lo que junto con la emersión del paradigma del desarrollo sostenible a nivel mundial motivó la aparición de la dimensión ambiental en los instrumentos de ordenamiento territorial de la RMBA.

\section{Propuestas y gestión histórica de las cuencas de la RMBA}

En Argentina, la dimensión ambiental en el planeamiento urbano no emerge hasta fines de la década de los 70. Uno de los primeros proyectos en tratar la problemática ambiental fue el proyecto para el SIMEB $^{5}$ (Sistema Metropolitano Bonaerense), el cual interpreta a la ciudad desde modelos ecosistémicos ya analizados desde otros ámbitos y disciplinas (CARIDE Bartrons, en Di Pacce, 2004); sin embargo, no es hasta el trabajo "Lineamientos estratégicos para la Región Metropolitana de Buenos Aires” (2007) cuando el enfoque del desarrollo sustentable se hace manifiesto en el tratamiento de los cursos de agua, incorporando en la matriz ambiental el manejo de cuencas y el espacio ribereño, el control de los rellenos, la gestión de los residuos, la protección de las reservas naturales, el control de las inundaciones y su inclusión dentro de la malla de áreas verdes (CAPUccio y MignaQui, 2014).

El interés a nivel nacional en el manejo de cuencas puede rastrearse en el informe "Problema Torrencial en Argentina” de 1950 (FAO), que sienta las bases para la institucionalización del tema a través del Instituto de Ordenación de Vertientes e Ingeniería Forestal (IOVIF), el cual se mantiene activo entre 1963 y 1975. Según la CEPAL (2005), a partir de la disolución de este organismo y hasta la década de 1990, predomina una visión de aprovechamiento del agua —que entiende que el manejo del recurso hídrico es necesario para la ocupación
5. El proyecto para el SIMEB es publicado en 1979 por el CONHABIT (Programa de

Concentración del hábitat y Ordenamiento Territorial). 
del territorio- acompañado de grandes obras hidráulicas. Bajo el paradigma del desarrollo sustentable, la cuenca comienza a presentarse como una unidad de análisis territorial donde interactúan aspectos biológicos, físicos y socioeconómicos (Di PACE y BARsKY, 2012).

En 2004 comienza la elaboración del Plan Estratégico Territorial (PET 2016), marco en el que se formulan los "Lineamientos Estratégicos Metropolitanos" (Subsecretaría de Urbanismo y Vivienda), en los que se incorpora decididamente el enfoque estratégico y ambiental en la gestión del territorio. Casi simultáneamente, en 2008, en la CABA se aprueba el Plan Urbano Ambiental (PUA), el cual considera el contexto metropolitano y la necesidad de coordinación interjurisdiccional. Sin embargo, la falta de una unidad de gestión territorial metropolitana y una autoridad que la coordine, así como la dispersión normativa dificultan la aplicación de instrumentos de ordenamiento territorial de enfoque ambiental. En este sentido, Pugliese y Sgroi (en Pintos y Narodowsky, 2012) sitúan tres abordajes o sistemas administrativos desde el punto de vista de la gestión territorial que debieran actuar integradamente en las urbanizaciones cerradas polderizadas del río Luján, enfoques que también deberían actuar integradamente en la gestión de cuencas de la RMBA: el urbano territorial, el ambiental y el de gestión de aguas.

Mientras el ordenamiento territorial de los municipios de la RMBA se rige por decreto Ley N. ${ }^{\circ} 8912 / 77$ y las normativas de ordenamiento urbano que cada municipio dicte en este marco - ya que estos son los que tienen la responsabilidad primaria del ordenamiento territorial-, la gestión ambiental se rige por la Ley 11.723/95 (Protección del Ambiente y los Recursos naturales), la Ley 12.257 (Código de Aguas de la Provincia de Buenos Aires) y por lo dictaminado por la Autoridad del Agua y la oficina para el Desarrollo Sostenible, y en la CABA las competencias ambientales pertenecen al Ministerio de Ambiente y Espacio Público (Capuccio y Mignaqui, 2014).

Dada la falta de una coordinación interjurisdiccional que supere las divisiones políticas y ordene el territorio desde un criterio y, por lo tanto, una delimitación ambiental, sumada a la dispersión de instrumentos normativos y a la falta de instrumentos de planificación de carácter vinculante, la gestión ambiental es dificultosa y se expresa claramente en la falta de gestión integral de las cuencas hidrográficas. Podría decirse que el caso más avanzado dentro de la región es la cuenca Matanza-Riachuelo, en la que un fallo histórico a favor de un grupo de vecinos que data del año 2008, dictado por la Corte Suprema de Justicia de la Nación, determinó los responsables 
de llevar a cabo las obras de saneamiento y la penalización en caso de incumplimiento de estas. Para el año 2006 ya se había sancionado la Ley 26.168, que creó la Autoridad de Cuenca Matanza-Riachuelo (ACUMAR), integrada por los tres niveles de gobierno que tienen injerencia en la cuenca (Nación, Provincia de Buenos Aires y Ciudad Autónoma de Buenos Aires y Municipiosº), con facultades de coordinación, ejecución y regulación y control.

En este marco se pone en marcha el Plan Integral de Saneamiento Ambiental (PISA7), actualizado en el año 2016, coordinado e implementado por la ACUMAR. Dentro del plan se aborda el eje ordenamiento ambiental del territorio, cuyo objetivo fundamental es establecer una "macro zonificación” (PISA, 2009: 357) que dé lugar a un crecimiento equilibrado y sustentable de la cuenca. El caso Matanza-Riachuelo es una referencia a nivel nacional por la importancia de la cuenca, así como por el avance del plan de saneamiento.

Siguiendo por las otras grandes cuencas de la región, en la cuenca del río Reconquista — que a diferencia de la cuenca de los ríos Matanza-Riachuelo, se encuentra íntegramente dentro de la provincia de Buenos Aires ${ }^{8}$ — desde mediados de la década de 1990, con la conformación del UNIREC, ${ }^{9}$ se realizan acciones de saneamiento, en el marco de un plan de saneamiento de la cuenca, hoy coordinadas por el Comité de la Cuenca Reconquista (COMIREC), creado en 2001 por Ley 12.653, que tiene como responsabilidad su planificación y ejecución. Esta cuenca cuenta con el "Programa de Gestión Urbano Ambiental Sostenible de la cuenca del río Reconquista” (financiado con préstamo BID aprobado en 2014).

Continuando por las otras cuencas metropolitanas, donde los cursos permanecen a cielo abierto, en las cuencas del río Luján y de la zona sur, los comités de cuenca ${ }^{10}$ se encuentran constituidos; sin embargo, no existen planes integrales de saneamiento.

Un caso en el que se materializan muchos de los temas planteados en relación con el proceso de ocupación del territorio y la gestión fragmentaria de las cuencas es en el Gran La Plata, microrregión donde el tema de los arroyos ganó interés desde la inundación ocurrida en abril de 2013. La cuenca del arroyo del Gato, ubicada entre los municipios de La Plata y Ensenada, fue una de las más afectadas, al tratarse de la cuenca más poblada de la microrregión.

A continuación se profundizan los temas planteados para la generalidad de las cuencas de la RMBA en el Gran La Plata y específicamente en la cuenca del arroyo del Gato.
6. Municipios de Almirante Brown, Avellaneda, Cañuelas, Esteban Echeverría, Ezeiza, General Las Heras, La Matanza, Lanús, Lomas de Zamora, Marcos Paz, Merlo y San Vicente.

7. Los objetivos del PISA (Plan Integral de Saneamiento Ambiental) son recomponer el ambiente, prevenir el daño y mejorar la calidad de vida (http://www.acumar. gov.ar).

8. La cuenca está integrada por dieciocho partidos:

Moreno, San Fernando, Tigre, Malvinas Argentinas, San Miguel, Hurlingham, Ituzaingó, Morón, San Isidro, General San Martín, Tres de Febrero, José C. Paz, San Fernando, General Las Heras, General Rodríguez, Luján, Marcos Paz y Merlo.

9. Unidad de Coordinación del Proyecto Río Reconquista.

10. Comité de Cuenca Río Luján (Resolución 03,

Autoridad del Agua, 2001); Comités de Cuenca Río de La Plata superior, intermedio e inferior (Resoluciones 189, 190 y 191, Autoridad del agua, 2008). 
11. Entre las influencias que guiaron el diseño

de la ciudad de La Plata

se ubica la corriente

higienista, desarrollada por sanitaristas ingleses

$y$ franceses a principios del siglo XIX. Se basa en

la preocupación por la salud de la ciudad y sus

habitantes, y trata de mantener determinadas condiciones de salubridad en el ambiente. En el diseño de la ciudad de La Plata tienen especial influencia las ideas de los sanitaristas Benjamin Ward Richardson y Edwin Chadwick (DE TERÁN y MoRosi, 1983).

\section{El Gran La Plata y la cuenca del arroyo del Gato}

Dentro de las cuencas de la zona sur, coincidiendo con la microrregión de La Plata, Berisso y Ensenada, existen diez cuencas pertenecientes a la vertiente del río de la Plata, integradas por más de quince arroyos. Dichas cuencas totalizan una superficie de $566.9 \mathrm{~km}^{2}$ (HURTADO ET ÁL., 2006), aproximadamente el $63 \%$ de la superficie del partido de la Plata $\left(893.53 \mathrm{~km}^{2}\right)$.

La ciudad de La Plata fue fundada sobre el paraje denominado en 1882 como "Lomas de Ensenada", parte de la pampa ondulada, con una altitud variable entre los 5 y los 22.50 msnm dentro del casco fundacional. Las lomas se sitúan por sobre los bañados, que desde la localidad de Bernal se extienden hacia el partido de La Plata, conformando la dimensión de una legua entre el Bosque y la isla Santiago (De Paula, 1987).

La fundación de la ciudad de La Plata en 1882, como capital de la provincia de Buenos Aires, dio lugar a profundas modificaciones del relieve natural, como sucedió en la ciudad de Buenos Aires y en el resto de las ciudades cabeceras de partido que hoy son parte de la región, donde no se consideró alterar el diseño de la retícula para evitar las planicies de inundación de los arroyos. Las ideas higienistas del siglo XIX ${ }^{11}$ concentraron el sistema de desagües pluviales sobre los principales cauces, siendo el entubamiento de los arroyos y sus afluentes una práctica constante desde el siglo XIX.

La Cuenca del Arroyo Del Gato (CADG) es la segunda de mayor extensión dentro del Gran La Plata, y la más poblada. Cuenta con una superficie de 12.400 ha, una población de 351.713 habitantes (FACULTAD DE InGENIERÍA, 2013), y recibe en su cauce principal la mayor proporción de desagües pluviales de la ciudad y localidades periféricas, junto con los arroyos Pérez y Regimiento, que funcionan como sus principales afluentes.

Se trata de la cuenca que ocupa la mayor superficie dentro del casco fundacional de la ciudad de La Plata, área en la que sus cursos se encuentran entubados, así como en algunos sectores periféricos. Sin embargo, el curso principal del arroyo discurre a cielo abierto casi en su totalidad.

A lo largo de la cuenca se dan diversas situaciones con respecto a los usos del suelo (figura 3), concentrándose tanto la urbanización intensiva como algunas de las concentraciones más relevantes de actividades de índole industrial y agropecuaria de la región. La cuenca 
Gestión de cuencas en la región Metropolitana de Buenos Aires. Historia y actualidad de un territorio en conflicto ambiental. El caso del Gran La Plata

alta es parte de la última actividad flori-hortícola de carácter intensivo de la región, en coexistencia con agricultura y ganadería extensivas, mientras que sobre la cuenca media se desarrolla una gran mixtura de usos, verificándose la mayor densidad residencial —con asentamientos precarios sobre las márgenes-, así como de actividades industriales y comerciales que conviven con algunos usos industriales y extractivos, activos e inactivos.

La cuenca baja está ubicada en la planicie costera/humedal, y el arroyo — canalizado- discurre dentro de ella y desemboca en el río Santiago, que se destaca por el paisaje natural del Monte Costero, declarado Paisaje Protegido por Ley Provincial N. ${ }^{0} 12756 .{ }^{12}$ Tempranamente las zonas aledañas al curso principal se ocuparon con usos industriales. Desde la fundación de La Plata en 1882 comenzaron a asentarse hornos de ladrillo, que definieron temprana-

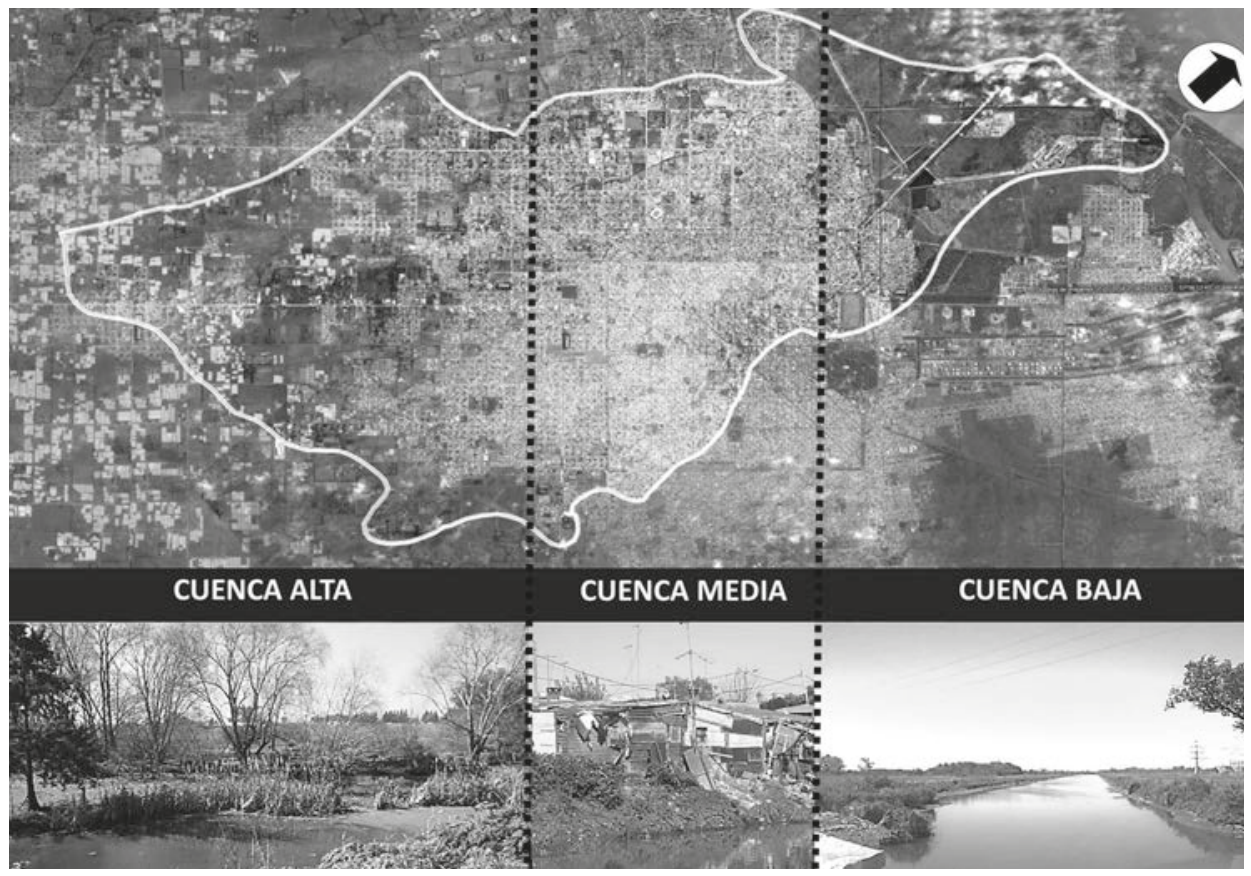

Figura 3.

Cuenca del arroyo del Gato. Tramos a lo largo del curso principal. Fuente: mapa de elaboración propia sobre la base de información de HURTADO ET ÁL., 2006 y Google Earth. Fotos del autor 
12. Paisaje Protegido de Interés Provincial para el desarrollo ecoturístico a la zona denominada Monte Ribereño Isla Paulino,

Isla Santiago. Senado y Cámara de Diputados de la Provincia de Buenos Aires. Año 2001.

13. Coordinación ecológica Área Metropolitana Sociedad del Estado.

14. Plan regulador conjunto de los partidos de La Plata y Ensenada (GRUPO URBIS, 1961).

15. Entre los estudios más relevantes cabe mencionar los siguientes:

Cartografía temática ambiental de la cuenca del arroyo del Gato, partidos

de La Plata y Ensenada, provincia de Buenos Aires

(CABRAL, GIMÉNEZ, SÁNCHEZ y CRINCOLI, 2005), Estudios Hidrológicos-HidráulicosAmbientales en la Cuenca del Arroyo del Gato

(ROMANAZZI Y URBIZTONDO, 2007) y Calidad ambiental de las Cuencas de los Arroyos del Gato y Pereyra (CIMA-UNLP, 2012). mente un perfil industrial que se consolidó en el período que va entre 1940 y 1980. La industrialización se vio acompañada por un crecimiento urbano por fuera del casco fundacional de La Plata, consolidó las localidades preexistentes y dio lugar a nuevas localizaciones en las periferias.

En este proceso la urbanización se extendió sobre la planicie de inundación del arroyo, y fueron ocupadas áreas inundables como los bañados y la antigua franja costera. La ocupación de estas últimas se intensificó durante el período actual, con la instalación del CEAM$\mathrm{SE}^{13}$ y la central termoeléctrica en el área de bañados y el crecimiento de los asentamientos precarios sobre las márgenes del arroyo. El crecimiento urbano a su vez va desplazando la producción agrícola y aumenta la producción bajo cubierta, lo que modifica las condiciones de escurrimiento de la cuenca y también el paisaje.

Desde el plano normativo, como antecedentes pueden reconocerse instrumentos regulatorios pertenecientes a los dos ámbitos jurisdiccionales (La Plata y Ensenada), que regulan las actividades en su cuenca. El Plan Regulador de $1961^{14}$ propone en gran parte de ella el establecimiento de una zona industrial para el partido de La Plata, aprovechando tanto la accesibilidad de los ejes de comunicación como el cauce del arroyo para las descargas industriales, obviando el valor ambiental y paisajístico del curso. Esta situación recién comienza a revertirse a partir de la década del 90, cuando se vislumbra desde los cuadros técnicos la retracción de la actividad industrial y la innecesaria dotación de tierra para esta actividad, junto con la importancia de estudiar las cuencas y conservar libres los márgenes de los cursos.

Además, la necesidad de contar con información del medio natural y antropizado para el tratamiento de las inundaciones llevó a la realización de estudios geológicos e hidrológicos de la cuenca ${ }^{15}$, trabajos que ponen énfasis en la situación crítica del recurso y en la necesidad de su tratamiento tanto con medidas estructurales como no estructurales. Las medidas estructurales agrupan a las construcciones que reducen o evitan el impacto de la inundación, incluyendo un amplio rango de obras de ingeniería civil. Su funcionalidad se encuentra limitada a eventos asociados a una cierta probabilidad de ocurrencia. En cambio, las medidas no estructurales incluyen el ordenamiento del territorio, el desarrollo del conocimiento, los mecanismos de participación pública, entre otras. Buscan la reducción de la vulnerabilidad de la población en riesgo a partir del planeamiento y la gestión llevados a cabo antes, durante y después de la catástrofe (Facultad de Arquitectura y Urbanismo, 2013). 
Se desarrollaron talleres durante los años 2012 y 2013, que fijaron los lineamientos de cara a la constitución de un "Plan de Gestión Integral de la cuenca del arroyo del Gato", impulsados por la Autoridad del Agua, OPDS y DIPSOH; sin embargo, el plan aún no se concretó. Las inundaciones de abril de 2013, además de otras de gran impacto como las de 2002, 2005 y 2008, en La Plata, Berisso y Ensenada, dejaron al descubierto la falta de planificación y gestión de las cuencas hidrográficas de la región. Las aguas, al ocupar la extensión de sus propias planicies de inundación y reocupar las huellas de los antiguos cauces, produjeron el anegamiento de amplias zonas urbanas (FACULTAD DE InGENIERÍA, 2013), lo que alertó a la población sobre su vulnerabilidad frente a la dinámica del medio natural sobre el cual la ciudad y su periferia se asientan.

Dichas inundaciones impulsaron obras de envergadura en la cuenca del arroyo como parte de un conjunto de obras planificadas y ejecutadas por la provincia de Buenos Aires para la región capital. En la cuenca del arroyo del Gato se planificaron y ejecutaron - parcial y totalmente- dos derivadores con el fin de reducir los caudales dentro del casco urbano, conduciéndolos hacia el curso principal del arroyo, en el cual se están realizando obras de ampliación del cauce, canalización y revestimiento en hormigón para facilitar el escurrimiento de la cuenca. Pero a pesar de ser la cuenca más estudiada de la región, no posee un plan de tratamiento ni fue incorporada adecuadamente a la ordenanza de usos del suelo, como parte de los instrumentos de ordenamiento territorial disponibles. Las intervenciones fragmentarias demuestran que no se reconoce a la cuenca como unidad de gestión.

El arroyo del Gato, por lo que representa a nivel regional, es un posible caso para poner a prueba estrategias de ordenamiento territorial cuyo objeto sea la mejora de la calidad ambiental, que podrían aplicarse a otras cuencas metropolitanas. Asimismo, representa una oportunidad en materia de paisaje, ya que el curso principal - a pesar del alto grado de ocupación que registra en muchos sectores- se encuentra abierto casi en su totalidad, y en algunas zonas el cauce posee escasa modificación.

\section{Reflexiones finales}

Desde la fundación de la ciudad de Buenos Aires como centro de la región, comienza a vislumbrarse un modelo de crecimiento urbano que ignora la dinámica natural de las cuencas hidrográficas. Tempranamente los cursos o sus orillas fueron emplazamientos de toda actividad o persona expulsada de la ciudad: ocupados desde el siglo XVII por sectores margina- 
les o de bajos recursos, emplazamiento de saladeros e industrias, con cada vez más escasas apariciones en la cartografía, hasta desaparecer totalmente cuando la tecnología empieza a permitir su transformación y la ciudad le gana al medio natural. Asimismo, desde lo simbólico-cultural los valores centrales de ríos y arroyos interiores se asocian a la desembocadura en el río de La Plata, sin que exista una apreciación sustancial de valores culturales y naturales en las cuencas media y alta.

Prematuramente parece imponerse la idea de que naturaleza y ciudad no son compatibles, o dicho de otra manera: el medio construido no puede convivir con el medio físico-natural. Los arroyos de la RMBA están asociados al imaginario de la periferia, y sus atributos resumen las ideas construidas históricamente en torno de este territorio, un espacio residual que condensa todo tipo de actividad contaminante y degradadora y donde la condición dominante es la informalidad en el uso y ocupación del suelo. Son espacios con una clara vocación funcional dentro de la estructura urbana, pero que no se asocian con la idea de ambiente como hecho socialmente valorado. Esta idea parece sostenerse hasta hoy, ya que se verifica en la gestión de cuencas hidrográficas en la RMBA, donde los enfoques urbano-territorial, ambiental y la gestión de las aguas actúan descoordinadamente.

Por ello, de cara a una gestión territorial de las cuencas en la RMBA que tome en cuenta la complejidad de este territorio en materia ambiental, es necesario ordenar la región sobre la base del estudio de los procesos ambientales, donde la regionalización con base en las cuencas sería la más adecuada no solo para la gestión del riesgo, sino para el tratamiento integral de los sistemas hídricos urbanos: las fuentes de agua, el almacenamiento y distribución, el saneamiento y el drenaje de las aguas -incluyendo el tratamiento de los residuos sólidos urbanos-, enfatizando la necesidad de mejorar la calidad paisajística de ríos y arroyos, como un modo de conservar la calidad ambiental.

En relación con la dimensión paisajística, la cuenca del arroyo del Gato, como muchas otras de la RMBA, conserva valores naturales relevantes en el marco de una región metropolitana: grandes superficies vacantes, bañados y bosques, el monte costero en la desembocadura, el curso abierto casi en su totalidad y en más de la mitad de su recorrido no revestido en hormigón. Asimismo la "artificialización” (Brailovsky, 2010) del medio natural en el casco ha provocado que las planicies de inundación de los cursos entubados de los arroyos Pérez y Regimiento sean las áreas con mayor altura de agua en calle durante la inundación de 2013. 
Es necesario poner en valor a la cuenca como unidad organizativa del territorio, al menos en dos sentidos. Por un lado, desde lo físico-natural, como unidad sintética de los procesos ambientales, siendo especialmente relevante para el análisis de un territorio dominantemente urbano como el de la RMBA, pero también como un tejido conectivo configurado a partir del agua y de los procesos ecológicos derivados de la interacción de los ámbitos terrestre y acuático, que desde lo físico-natural vincula los fragmentos territoriales que configuran la región, siendo el elemento que desde el medio natural mejor resume las relaciones entre ambiente y cultura que se dan en la Región Metropolitana de Buenos Aires.

\section{Bibliografía}

ACUMAR (Autoridad de Cuenca Matanza Riachuelo) (2009) "Plan Integral de Saneamiento Ambiental". Recuperado de http://www.acumar.gob.ar/.

BRAILOVSKY, Antonio Elio (2010) Buenos Aires, ciudad inundable. Por qué está condenada a un desastre permanente. Capital Intelectual, Buenos Aires.

CABRAL, Mirta Graciela; GIMÉNEZ, Jorge Eloy; SÁNCHEZ, Carlos Ariel y CRINCOLI, Alina (2005). "Cartografía temática ambiental de la cuenca del arroyo del Gato, partidos de La Plata y Ensenada, provincia de Buenos Aires”. CISAUA: Instituto de Geomorfología y Suelos, La Plata.

CAPUCCIO, Silvana y MIGNAQUI, Iliana (2014). "Paradigmas Urbanísticos y Gestión Ambiental Metropolitana. Aportes de la Planificación Ecológica y sus posibilidades de aplicación en la Región Metropolitana de Buenos Aires”. 11. ${ }^{\circ}$ Simposio de la Asociación Internacional de Planificación Urbana y Ambiente (UPE 11). Universidad Nacional de La Plata, La Plata.

CENTRO DE INVESTIGACIÓN DEL MEDIO AMBIENTE CIMA-UNLP (2012). "Calidad ambiental de las Cuencas de los Arroyos del Gato y Pereyra”. Informe final proyecto PNUD-FREPLATA. Recuperado de http://www.ambiente.gob.ar/.

CEPAL (2005). "Entidades de gestión del agua a nivel de cuencas: experiencia de Argentina”. Publicación de las Naciones Unidas, Santiago de Chile.

DE PAULA, Alberto (1987) La ciudad de La Plata, sus tierras y su arquitectura. Ed. Banco Provincia de Buenos Aires, Buenos Aires.

DE TERÁN, Fernando y MOROSI, Julio (coords.) (1983) La Plata: ciudad nueva ciudad antigua. Historia, forma y estructura de un espacio urbano singular. Editorial del Instituto de Estudios de Administración Local, Madrid.

DI PACE, María y CARIDE, Horacio (Eds.) (2004) Ecología de la Ciudad. Prometeo, Buenos Aires. 
DI PACE, María y BARSKY, Andrés (Dir.) (2012) Agua y territorio. Fragmentación y complejidad en la gestión del recurso hídrico en la Región Metropolitana de Buenos Aires. Ciccus, Buenos Aires.

ETULAIN, Juan Carlos y LÓPEZ, Isabel (1999). "El crecimiento de la metrópolis. La RMBA en el escenario de la reestructuración global”. V Seminario Internacional de la Red Iberoamericana de Investigadores (RII), sobre Globalización y Territorio. Universidad Autónoma del Estado de México, Toluca, México.

FACULTAD DE ARQUITECTURA Y URBANISMO, UNIVERSIDAD NACIONAL DE LA PLATA (2013). “Concurso Latinoamericano de estudiantes de arquitectura Repensar La Plata, Ideas para la cuenca del arroyo Del Gato. Región Gran La Plata, Argentina”. Bases. Disponible en http://www.fau.unlp.edu.ar/shared_resource/pdf/html/bases-concurso-repensar.pdf. FACULTAD DE INGENIERÍA (2013). "Estudio sobre la inundación ocurrida los días 2 y 3 de abril de 2013 en las ciudades de La Plata, Berisso y Ensenada”. Recuperado de http://sedici. unlp.edu.ar/handle/10915/27334.

GRUPO URBIS (1961). "Plan regulador conjunto de los partidos de La Plata y Ensenada”. Cap. IV, V, VI, VIII. Municipalidad de La Plata, La Plata.

GUTMAN, Margarita y HARDOY, Jorge Enrique (2007) Buenos Aires 1536-2006. Historia urbana del área metropolitana. Ediciones Infinito, Buenos Aires.

HERRERO, Ana Carolina y FERNÁNDEZ, Leonardo (2008) De los ríos no me río: diagnóstico y reflexiones sobre las Cuencas Metropolitanas. Temas Grupo Editorial, Buenos Aires.

HURTADO, Martín; GIMÉNEZ, Jorge; CABRAL, Mirta; SILVA, Mario; MARTÍNEZ Omar; CAMILIÓN, María Cecilia... LUCESOLI, Horacio (2006) Análisis ambiental del partido de La Plata. Aportes al ordenamiento territorial. Consejo Federal de Inversiones-Municipalidad de la Plata, La Plata.

PINTOS, Patricia y NARODOWSKI, Patricio (Coords.) (2012) La privatopía sacrílega. Efectos del urbanismo privado en humedales de la cuenca baja del río Luján. Imago Mundi, Buenos Aires. ROMANAZZI, Pablo y URBIZTONDO, Arturo (2007). "Estudios Hidrológicos-Hidráulicos-Ambientales en la Cuenca del Arroyo del Gato. Informe Final”. Laboratorio de Hidrología, Área Departamental Hidráulica, Facultad de Ingeniería. Universidad Nacional de La Plata, La Plata. SILVESTRI, Graciela (2003) El color del río. Historia cultural del paisaje del Riachuelo. Prometeo, Buenos Aires.

SUBSECRETARÍA DE URBANISMO Y VIVIENDA-DPOUT (2007). "Lineamientos Estratégicos para la Región Metropolitana de Buenos Aires”. Ministerio de Obras Públicas de la Provincia de Buenos Aires, Buenos Aires. 